\title{
MOTIFSIM: A web tool for detecting similarity in multiple DNA motif datasets
}

\author{
Ngoc Tam L. Tran* and Chun-Hsi Huang \\ Department of Computer Science and Engineering, University of Connecticut, Storrs, CT
}

BioTechniques 59:26-33 (July 2015) doi 10.2144/000114308

Keywords: transcription factor binding site; DNA motif; motif detection tool; motif similarity

Supplementary material for this article is available at www.BioTechniques.com/article/114308.

Currently, there are a number of motif detection tools available that possess unique functionality. These tools often report different motifs, and therefore use of multiple tools is generally advised since common motifs reported by multiple tools are more likely to be biologically significant. However, results produced by these different tools need to be compared and existing similarity detection tools only allow comparison between two data sets. Here, we describe a motif similarity detection tool (MOTIFSIM) possessing a web-based, user-friendly interface that is capable of detecting similarity from multiple DNA motif data sets concurrently. Results can either be viewed online or downloaded. Users may also download and run MOTIFSIM as a command-line tool in stand-alone mode. The web tool, along with its command-line version, user manuals, and source codes, are freely available at http://biogrid-head.engr.uconn.edu/motifsim/.

Several computational tools have been developed to detect binding site motifs. Previous work showed that results reported by different motif finding tools from the same data set may vary significantly (1). This is largely due to the fact that different tools adopt different strategies that may be tailored for different motif groups. It is generally advisable to use multiple tools on the same data set as motifs commonly reported by different tools are more likely to be biologically significant (1). However, the results reported by multiple tools from the same data set need to be compared with each other in order to identify motifs detected by all of the tools, as well as those reported by some tools but not by others. Existing tools and methods described in the literature for finding motif similarity include STAMP (2), TOMTOM (3), MATLIGN (4), a method developed by Habib et al. (5), CompariMotif (6) and a method developed by Xu et al. (7), among a few others. These tools do not allow comparison of more than two motif data sets concurrently to extract common significant motifs. Instead, they only allow motif comparisons within a data set or between two data sets. In order to make motif comparisons in more than two data sets, pair-wise comparisons are performed first, and the results are then checked against each other manually, a lengthy and time-consuming process. Therefore, we developed a novel algorithm and implemented it as a web tool called MOTIFSIM to automate this process. A command-line version of the tool that runs in the stand-alone mode is also available for users to download. To the best of our knowledge, this is the first web tool that allows detection of similarity in multiple DNA motif data sets simultaneously.

\section{Materials and methods}

The motif representation format used in this study is the position-specific probability matrix (8). MOTIFSIM adopts a matrix of $I \times 4$ with $/$ rows and 4 columns, where $/$ represents the motif length and the 4 columns represent the A, C, G, and T nucleotides. Each entry in this matrix is the probability value of a nucleotide. The sum of the 4 values associated with nucleotides A, C, G, and T in each row must be 1. An illustration of a motif represented in the position-specific probability matrix is shown in Supplementary Figure S1. MOTIFSIM converts motifs in various formats from different motif finding tools to position-specific probability matrices, calculates their reverse complements, and combines them into one list. The procedures for converting various motif input formats to position-specific probability matrices are described in the Supplementary Material.

To detect similarity between the tools' motif predictions, MOTIFSIM performs

\section{METHOD SUMMARY}

To find similarity in multiple DNA motif datasets, researchers usually have to perform pair-wise comparisons by a motif similarity detection tool and then manually check the results from all of the pair-wise comparisons against each other. The MOTIFSIM web tool was designed to automate this process and provide bioinformatics researchers with a simple user-friendly and efficient tool for the analysis. The web tool is scalable as web traffic is balanced among multiple processors via a load balancer, and the computing module is multithreaded to allow efficient resource utilization. 


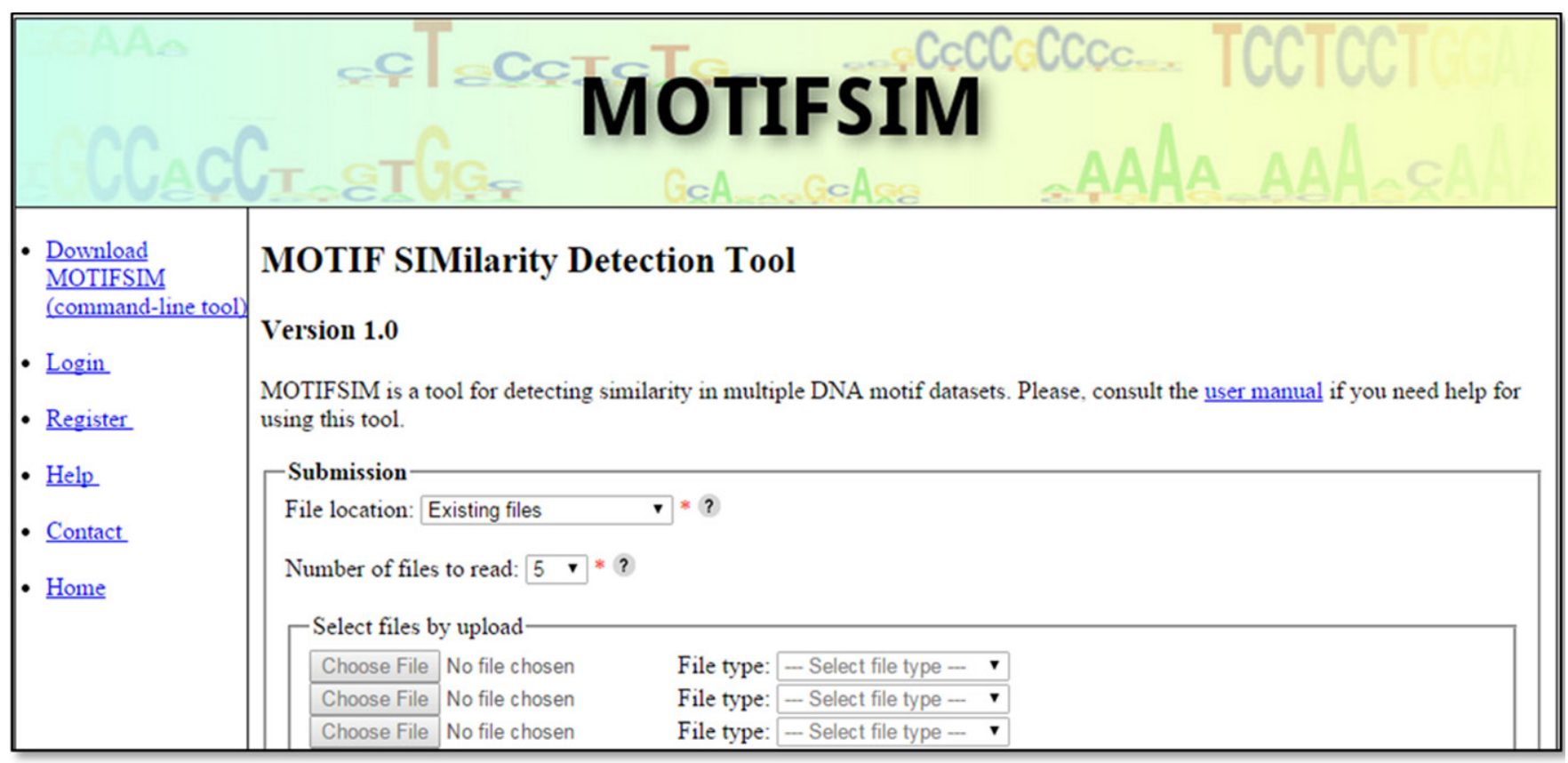

\begin{tabular}{|l|ll|l||}
\hline & {$\left[\begin{array}{lll|l|}\hline \text { Select existing files } & \\
\hline \text { DREME_DM230.txt } & \text { File type: } & \text { MEME output } \\
\hline \text { MEME_DM230.txt } & \text { File type: } & \text { MEME output } \\
\hline \text { PScanChIP_DM230.txt } & \text { File type: } & \text { Jaspar } \\
\hline \text { RSAT_peak-motifs_DM230.txt } & \text { File type: } & \text { TRANSFAC-like } \\
\hline \text { W-ChIPMotifs_DM230.txt } & \text { File type: } & \text { PSSM } \\
\hline\end{array}\right.$} \\
\hline
\end{tabular}

\begin{tabular}{|l|l||}
\hline & $\begin{array}{l}\text { Number of best matches (between 1 and 50): } 10 \\
\text { Cutoff (in percentage) for similarity }(\geq): 75 \mathbf{7 5} * ? \\
\text { Submit } \\
\text { * Required field. }\end{array}$ \\
\hline
\end{tabular}

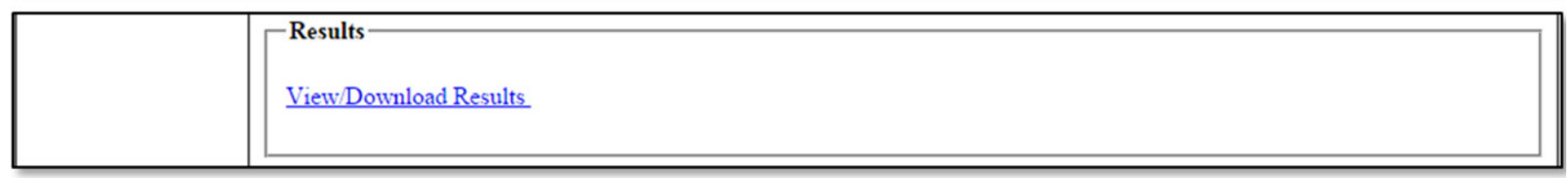

Figure 1. MOTIFSIM web interface. Portion of MOTIFSIM web interface showing an example comparison of 5 existing motif data sets with the 10 best matches selected using $75 \%$ for the similarity cutoff.

pair-wise comparisons on the entire motif list in matrix format. The pair-wise comparison is carried out by performing element-to-element comparisons for the entire overlapping window between two matrices. The maximum overlapping window can be the size of the matrix, while the minimum overlapping window is limited to four columns. The forward alignment begins at the first row on the top and progressively shifts downward, while the backward alignment begins at the last row at the bottom and progressively shifts upward. For each position in both forward and backward alignments including their reverse complements, a similarity score between two matrices is calculated using the element-to-element comparison approach.

\section{Algorithm}

Our novel algorithm comprises the following steps:

1. Combine motifs from multiple data sets with different formats into one list $M$. In this step, motifs from $n$ input data sets in different formats are converted to position-specific probability matrices and combined into one list $M$. Their reverse complements are also calculated for comparisons.
2. Perform pair-wise comparisons on the entire list $M$.

This step performs forward and backward comparisons on each pair of the matrices, including their reverse complements, in $M$ as follows:

For each matrix $p_{i}$ ( $i$ from 1 to $m$ ) in $M$ (where $m$ is the number of matrices in the combined list $M$ ), perform forward and backward pair-wise comparisons on:

(i) $p_{i}$ with $p_{i+1}, p_{i+2}, p_{i+3}, \ldots, p_{m}$.

(ii) $p_{i}$ reverse complement with $p_{i+1}, p_{i+2}, p_{i+3}, \ldots, p_{m}$. 
Table 1. Comparison of global significant motifs identified by MOTIFSIM with motif results reported by STAMP for ChIP-Seq data set DM230.

\begin{tabular}{|c|c|c|c|c|}
\hline Motif name & Motif's logo & $\begin{array}{l}\text { UniProbe database (mouse) } \\
\text { match }\end{array}$ & $\begin{array}{l}\text { MOTIFSIM (number of tools and } \\
\text { the tools' names) }\end{array}$ & $\begin{array}{c}\text { STAMP (number of tools and } \\
\text { the tools' names) }\end{array}$ \\
\hline wbgTAAATAww & ${ }^{2} \cdot \ldots$ & Foxa2 & $\begin{array}{l}4 \\
\text { MEME } \\
\text { PScanChIP } \\
\text { RSAT_peak-motifs } \\
\text { W-ChIPMotifs }\end{array}$ & $\begin{array}{l}2 \\
\text { MEME } \\
\text { RSAT_peak-motifs }\end{array}$ \\
\hline SP1 & & SP1 & $\begin{array}{l}4 \\
\text { MEME } \\
\text { PScanChIP } \\
\text { RSAT_peak-motifs } \\
\text { W-ChIPMotifs }\end{array}$ & $\begin{array}{l}5 \\
\text { DREME } \\
\text { MEME } \\
\text { PScanChIP } \\
\text { RSAT_peak-motifs } \\
\text { W-ChIPMotifs }\end{array}$ \\
\hline Motif 21 & & Smad3_secondary & $\begin{array}{l}5 \\
\text { DREME } \\
\text { MEME } \\
\text { PScanChIP } \\
\text { RSAT_peak-motifs } \\
\text { W-ChIPMotifs }\end{array}$ & $\begin{array}{l}5 \\
\text { DREME } \\
\text { MEME } \\
\text { PScanChIP } \\
\text { RSAT_peak-motifs } \\
\text { W-ChIPMotifs }\end{array}$ \\
\hline ArntAhr & ${ }^{2}+$ & ArntAhr & $\begin{array}{l}5 \\
\text { DREME } \\
\text { MEME } \\
\text { PScanChIP } \\
\text { RSAT_peak-motifs } \\
\text { W-ChIPMotifs }\end{array}$ & $\begin{array}{l}4 \\
\text { DREME } \\
\text { PScanChIP } \\
\text { RSAT_peak-motifs } \\
\text { W-ChIPMotifs }\end{array}$ \\
\hline TFW1 & & $\mathrm{E} 2 \mathrm{~F} 1$ & $\begin{array}{l}5 \\
\text { DREME } \\
\text { MEME } \\
\text { PScanChIP } \\
\text { RSAT_peak-motifs } \\
\text { W-ChIPMotifs }\end{array}$ & $\begin{array}{l}4 \\
\text { DREME } \\
\text { PScanChIP } \\
\text { RSAT_peak-motifs } \\
\text { W-ChIPMotifs }\end{array}$ \\
\hline TFW2 & 2 & Myc & $\begin{array}{l}4 \\
\text { MEME } \\
\text { PScanChIP } \\
\text { RSAT_peak-motifs } \\
\text { W-ChIPMotifs }\end{array}$ & $\begin{array}{l}5 \\
\text { DREME } \\
\text { MEME } \\
\text { PScanChIP } \\
\text { RSAT_peak-motifs } \\
\text { W-ChIPMotifs }\end{array}$ \\
\hline HIFIAARNT & ${ }_{0}^{2}=$ & HIF1AARNT & $\begin{array}{l}4 \\
\text { MEME } \\
\text { PScanChIP } \\
\text { RSAT_peak-motifs } \\
\text { W-ChIPMotifs }\end{array}$ & $\begin{array}{l}2 \\
\text { PScanChIP } \\
\text { RSAT_peak-motifs }\end{array}$ \\
\hline TFAP2A & & TFAP2A & $\begin{array}{l}4 \\
\text { MEME } \\
\text { PScanChIP } \\
\text { RSAT_peak-motifs } \\
\text { W-ChIPMotifs }\end{array}$ & $\begin{array}{l}4 \\
\text { DREME } \\
\text { PScanChIP } \\
\text { RSAT_peak-motifs } \\
\text { W-ChIPMotifs }\end{array}$ \\
\hline wwAAATAATAtw & ${ }_{0}^{2}=$ & Glis2_secondary & $\begin{array}{l}3 \\
\text { MEME } \\
\text { RSAT_peak-motifs } \\
\text { W-ChIPMotifs }\end{array}$ & $\begin{array}{l}3 \\
\text { MEME } \\
\text { RSAT_peak-motifs } \\
\text { W-ChIPMotifs }\end{array}$ \\
\hline tkAAATAATAtw & : A A & Glis2_secondary & $\begin{array}{l}3 \\
\text { MEME } \\
\text { RSAT_peak-motifs } \\
\text { W-ChIPMotifs }\end{array}$ & $\begin{array}{l}3 \\
\text { MEME } \\
\text { RSAT_peak-motifs } \\
\text { W-ChIPMotifs }\end{array}$ \\
\hline
\end{tabular}

(iil) $p_{i}$ with $p_{i+1}$ reverse complement, $p_{i+2}$ reverse complement, $p_{i+3}$ reverse complement, ..., $p_{m}$ reverse complement.

(iv) $p_{i}$ reverse complement with $p_{i+1}$ reverse complement, $p_{i+2}$ reverse complement, $p_{i+3}$ reverse complement, $\ldots, p_{m}$ reverse complement.

3. Calculate similarity score between two motifs.

The similarity score between two matrices in Step 2 is calculated as follows:
For each position in Steps 2.i-2.iv, for each pair of matrices $\left(p_{i}, p_{i+1}\right)$ in a pair-wise comparison:

(i) Calculate the absolute value of the difference between each pair of corresponding matrix elements

$p_{i_{j, k} \text { and }} P_{(i+1)_{j, k}}$

in the overlapping window between two matrices

$P_{i \text { and }} P_{(i+1) \text {. }}$
The details for calculating this absolute value of the difference

$d_{(i, i+1)_{j, k}}\left(p_{i_{j, k}}, p(i+1)_{j, k}\right)$

can be found in the Supplementary Material.

(ii) Calculatetheaverage of the differences for each overlapping window between two matrices.

The complement of this value is the similarity $S_{(i, j+1)}$ between two matrices 
Table 2. Comparison of global significant motifs identified by MOTIFSIM with motif results reported by STAMP for ChIP-Seq data set DM05.

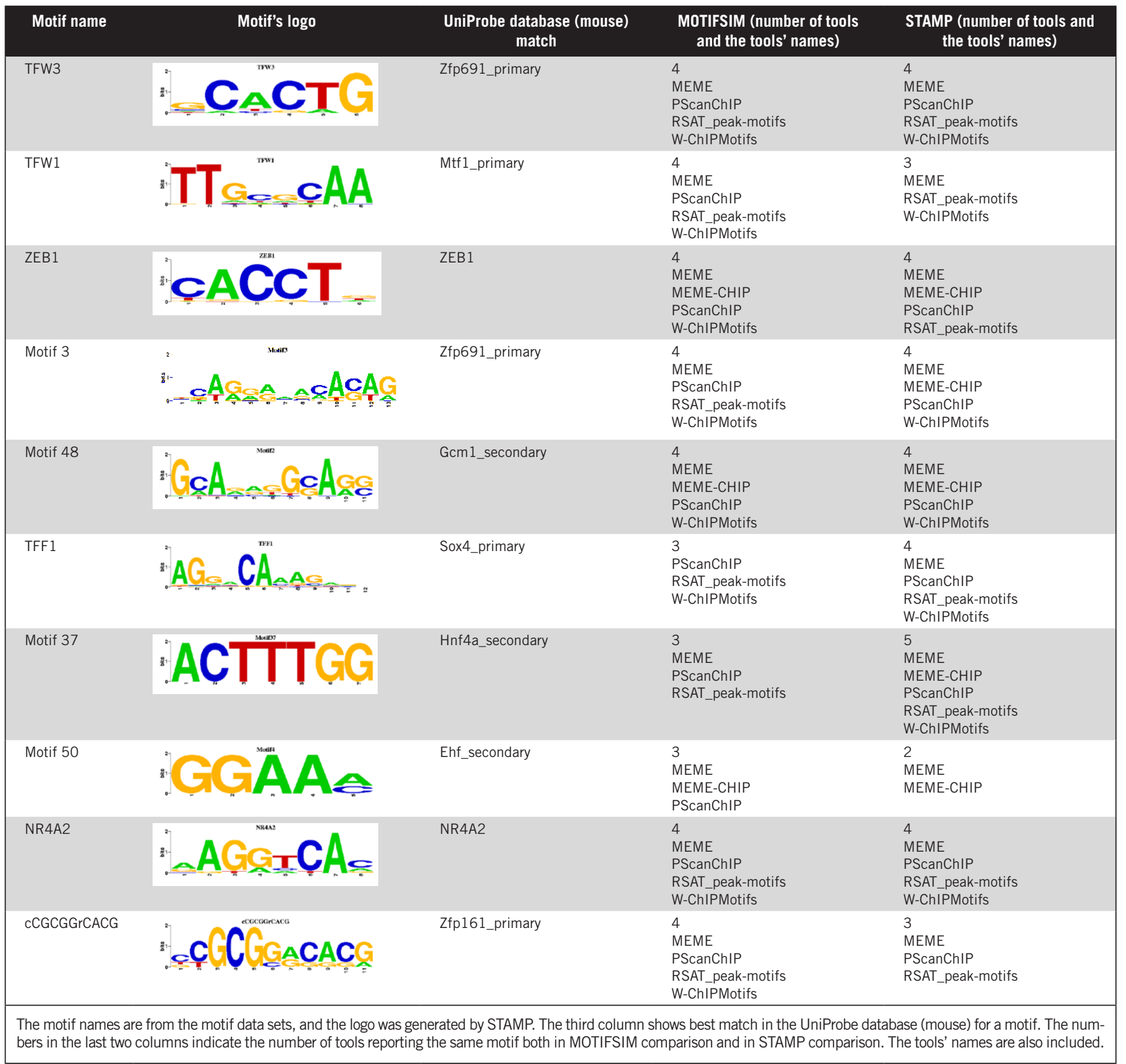

\section{Implementation}

The front-end of our web tool was implemented in HTML and JavaScript. The back-end was implemented in PHP, SQL, and $\mathrm{C}++$ with OpenMP for the purpose of multithreading. We used MySQL server for the back-end database to keep track of registered users. The web tool was deployed on a Linux cluster server with load balancing. Web traffic is directed to different Apache web servers on the cluster through the HAProxy (High Availability Proxy) load balancer (HAProxy - The Reliable, High Performance TCP/HTTP Load Balancer; available at http://www.haproxy.org). Supplementary Figure S2 shows the network architecture of the MOTIFSIM web tool.

HAProxy is a fast, reliable, high performance, and open source TCP/ HTTP load balancer. We use HAProxy's Round Robin load balancing algorithm for alternating web traffic to different nodes. HAProxy also performs health checks periodically via TCP connections to back-end nodes before forwarding the traffic. If a node fails the health check, web traffic is not forwarded to this node until it becomes healthy again.

\section{Results and discussion}

Usage

Motif data sets generated by various motif detection tools are fed into MOTIFSIM for comparison. The web tool can be used with or without user registration. Registered users can store the data sets and results on the web site for an extended period. Users can also upload data sets or use existing data sets for similarity detection. Currently, the web tool allows comparing up to ten motif data sets simultaneously.

\section{Input Formats}

The web tool accepts various input formats, including TRANSFAC (9), 
Table 3. Comparison of global significant motifs identified by MOTIFSIM with motif results reported by STAMP for ChIP-Seq data set DM721.

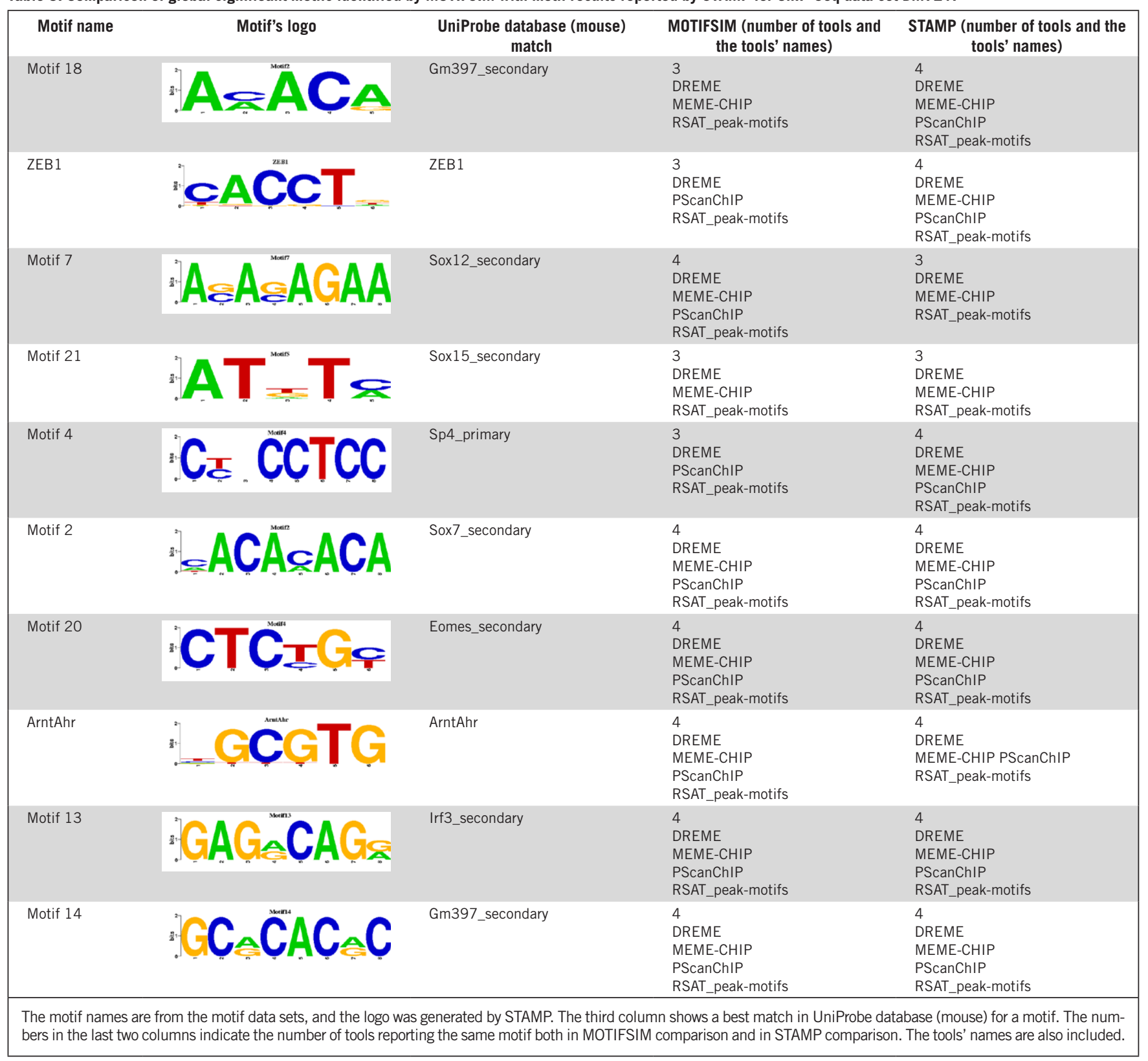

TRANSFAC-like (2), Position-Specific Scoring Matrix (PSSM), Jaspar (10), MEME's output (11), consensus sequence (2), sequence alignment (2), and matrices in horizontal or vertical formats. Details and examples of each input format are described in the user manual available from the web site.

\section{Input Parameters}

The web tool requires specifying the file type in use (uploaded by users, or existing files), number of input files, file format, number of best matches, and similarity cutoff in percentage. Further details of each input parameter can be found in the user manual.

\section{Outputs}

The web tool generates the results in two text files, where one includes motif details as position-specific probability matrices and the other does not. Each result file has Input and Results sections. The Input section contains input parameters and properties of each data set. The Results section includes three subsections for reporting global significant motifs, global and local significant motifs, and best matches for each motif, respectively. Details of the output file are also described in the user manual. The results can be viewed directly from the browser or can be downloaded for off-line use.
Figure 1 shows the MOTIFSIM web interface where users can upload or use existing input files to run the tool. The figure shows an example comparison of five existing motif files: MEME_DM230.txt in MEME output format, DREME_DM230.txt in MEME output format, PScanChIP_DM230.txt in Jaspar format, RSAT_peak-motifs_ DM230.txt in TRANSFAC-like format, and W-ChIPMotifs_DM230.txt in PSSM format (1). These files were generated by the MEME (11), DREME (12), PScanChIP (13), RSAT peak-motifs (14), and W-ChIPMotifs (15) motif detection tools, respectively, for the same ChIP-Seq data set, DM230, listed in Supple- 
mentary Table S1 (1). The selected number of best matches was 10, and the cutoff for similarity was $75 \%$. The results can be viewed or downloaded via the link at the bottom of the page. The input files and their comparison results can also be found in the sections Download Test Data sets and Download Sample Test Results in the user manual on the web site. We also have provided several other test data sets as well as sample test results for download or online viewing. They can be found via links in the user manual. Users can also contact us directly via the Contact page on the web site.

\section{Case Studies}

We evaluated MOTIFSIM on several motif data sets generated by MEME, DREME, PScanChIP, RSAT peak-motifs, and W-ChIPMotifs for each ChIP-Seq data set in Supplementary Table S1. The motif data sets reported by different tools for the ChIP-Seq data set used in each case study can be found in Supplementary Table S2. In each case study, we compared the global significant motifs identified by MOTIFSIM with the motif results generated by STAMP (2), using pair-wise comparison on each pair of motif data sets. The advantage of using STAMP is that it accepts motif data sets from various formats. However, STAMP can only compare two data sets at a time.

Case Study 1: ChIP-Seq Data set DM230 for Pol II (RNA polymerase II) We compared five motif data sets generated by five different tools in Supplementary Table S2 for the ChIP-Seq data set DM230 using MOTIFSIM. We selected the 10 best matches and used $75 \%$ for the similarity cutoff in this experiment. STAMP was used to perform pair-wise comparisons on these 5 motif data sets with the filters for $E$-value set to be $\leq 0.05$ and the number of best matches set at 10. For each global significant motif found by MOTIFSIM, we performed a manual lookup to find which tool reported it using the results from pair-wise comparisons. Table 1 also shows the tools reporting the same motif reported both in the MOTIFSIM comparison and the STAMP comparison. The motif name came from the motif data sets, and the logo was generated by STAMP. The third column shows a best match for the motif in the UniProbe database (16) for mouse. The numbers in the last two columns indicate the number of tools reporting the same motif in MOTIFSIM comparison and in STAMP comparison. The results show that all global significant motifs identified by MOTIFSIM were also discovered by STAMP using pair-wise comparisons. These motifs were reported by multiple tools in both MOTIFSIM and STAMP comparisons. We matched the tools reporting the same motif in both MOTIFSIM and STAMP and observed an $83 \%$ match between MOTIFSIM and STAMP.

Case Study 2: ChIP-Seq Data set DM05 for 0300 (co-activator protein) We repeated the procedure described in Case Study 1 for five motif data sets detected for the ChIP-Seq data set DM05. The results in Table 2 show an 85\% match between MOTIFSIM and STAMP.

\section{Case Study 3: ChIP-Seq Data set DM721} for H3K27ac (H3 lysine 27 acetylation) We repeated the procedure described in Case Study 1 for four motif data sets

Sometimes it takes more than one slice to make a great sandwich.

\section{The SageELF ${ }^{\mathrm{TM}}$ from Sage Science.}

\section{Collect multiple slices from a single sample.}

\section{Automated Gel Purification for: \\ - Next Gen Sequencing \\ - Protein Mass Spec}

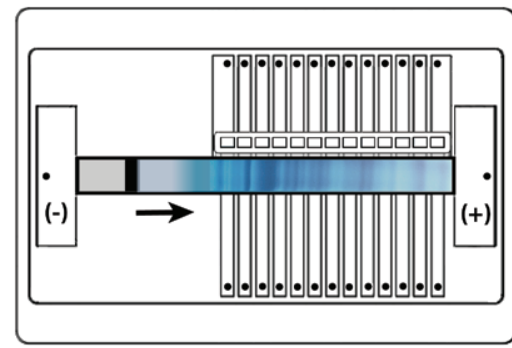

Separation

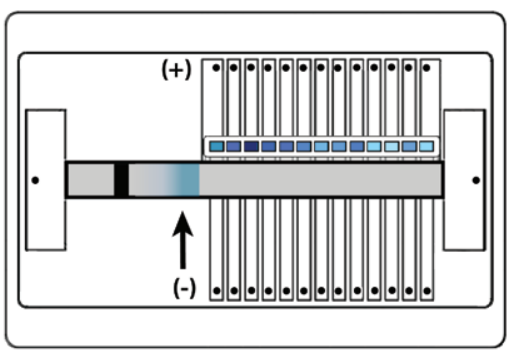

Fractionation
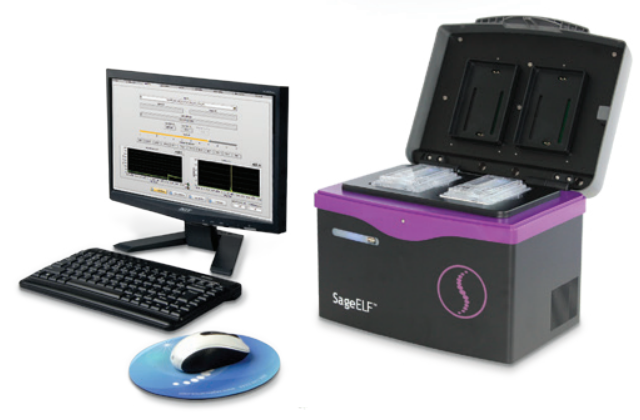
detected for the ChIP-Seq data set DM721. The comparison results can be found in Table 3, which shows a 90\% match between MOTIFSIM and STAMP.

Using multiple tools for finding motifs has the advantage for discovering motifs that a single tool may miss. In addition, motifs reported by multiple tools are more likely to be biologically significant. However, in the past there was no tool for identifying these motifs automatically. With MOTIFSIM, these motifs now can be easily identified automatically as global significant motifs. The results in the case studies above show MOTIFSIM is efficient for identifying common significant motifs in multiple data sets. Moreover, MOTIFSIM is able to identify similar significant motifs within a data set as well as in other data sets and report them as global and local significant motifs. MOTIFSIM also further identifies best matches in the combined list for every motif in every data set. Future development of the web tool includes the employment of a cloud computing platform to provide even more powerful computing services for larger motif data generated using next-generation sequencing (NGS) technology and additional storage space for storing uploaded data sets and the results.

The MOTIFSIM web tool is simple and easy to use. Without MOTIFSIM, finding similarity in multiple DNA motif data sets is a time-consuming process that requires pair-wise comparisons by a motif similarity detection tool such as STAMP. Results from all of the pair-wise comparisons then need to be manually checked against each other. Our web tool was designed to automate this process and provide bioinformatics researchers with a user-friendly and efficient tool for the analysis. Moreover, the web tool is scalable as web traffic is balanced via the HAProxy load balancer and the compute kernel is multithreaded to allow efficient resource utilization.

\section{Author contributions}

N.T.L.T. designed the algorithm, built the web tool and its command-line version, performed the case studies, and drafted the manuscript. C.-H.H. directed the research and helped to draft the manuscript. Both authors read and approved the final manuscript.

\section{Acknowledgments}

The authors would like to thank the anonymous reviewers for their comments and suggestions, which helped to improve this paper. This work was supported in part by the National Science Foundation (NSF) (Grant OCl-1156837 to C.-H.H.) and by U.S. Department of Education Graduate Fellowships in Areas of National Need (GAANNs) (Grant P200A130153 to N.T.L.T.).

\section{Competing interests}

The authors declare no competing interests.

\section{References}

1. Tran, N.T. and C.-H. Huang. 2014. A survey of motif finding Web tools for detecting binding site motifs in ChIP-Seq data. Biol. Direct 9:4

2. Mahony, S. and P. Benos. 2007. STAMP: a web tool for exploring DNA-binding motif similarities. Nucleic Acids Res. 35:W253-W258.

3. Gupta, S., J.A. Stamatoyannopoulos, T.L. Bailey, and W.S. Noble. 2007. Quantifying similarity between motifs. Genome Biol. 8:R24.

4. Kankainen, M. and A. Loytynoja. 2007. MATLIGN: a motif clustering, comparison and matching tool. BMC Bioinformatics 8:189.

5. Habib, N., T. Kaplan, H. Margalit, and N. Friedman. 2008. A Novel Bayesian DNA Motif Comparison Method for Clustering and Retrieval. PLOS Comput. Biol. 4:e1000010.

6. Edwards, R.J., N.E. Davey, and D.C. Shields. 2008. CompariMotif: quick and easy comparisons of sequence motifs. Bioinformatics 24:1307-1309.

7. Xu, M. and Z. Su. 2010. A Novel AlignmentFree Method for Comparing Transcription Factor Binding Site Motifs. PLoS ONE 5:e8797.

8. Li, H. 2002. Computational approaches to identifying transcription factor binding sites in yeast genome. Methods Enzymol. Part B 350:484-495.

9. Matys, V., E. Fricke, R. Geffers, E. Gossling, M. Haubrock, R. Hehl, K. Hornischer, D. Karas, et al. 2003. TRANSFAC@: transcriptional regulation, from patterns to profiles. Nucleic Acids Res. 31:374-378.

10. Sandelin, A., W. Alkema, P. Engstrom, W.W. Wasserman, and B. Lenhard. 2004. JASPAR: an open-access database for eukaryotic transcription factor binding profiles. Nucleic Acids Res. 32:D91-D94.

11. Bailey, T.L., N. Williams, C. Misleh, and W. Li. 2006. MEME: discovering and analyzing DNA and protein sequence motifs. Nucleic Acids Res. 34:W369-W373.

12. Bailey, T.L. 2011. DREME: motif discovery in transcription factor ChIP-seq data. Bioinformatics 27:1653-1659.

13. Zambelli, F., G. Pesole, and G. Pavesi. 2013. PscanChIP: finding over-represented transcription factor-binding site motifs and their correlations in sequences from ChIP-Seq experiments. Nucleic Acids Res. 41:W535W543.

14. Thomas-Chollier, M., C. Herrmann, M. Defrance, O. Sand, D. Thieffry, and J. van Helden. 2012. RSAT peak-motifs: motif analysis in full-size ChIP-seq datasets. Nucleic Acids Res. 40:e31.

15. Jin, V.X., J. Apostolos, N.S. Nagisetty, and P.J. Farnham. 2009. W-ChIPMotifs: a web application tool for de novo motif discovery from ChIP-based high-throughput data. Bioinformatics 25:3191-3193.

16. Newburger, D.E. and M. Bulyk. 2009. UniPROBE: an online database of protein binding microarray data on protein-DNA interactions. Nucleic Acids Res. 37:D77-D82.

Received 23 January 2015; accepted 04 May 2015.

Address correspondence to Ngoc Tam L. Tran, Department of Computer Science and Engineering, University of Connecticut, Storrs, CT. E-mail: ntt10001@engr.uconn.edu

To purchase reprints of this article, contact: biotechniques@fosterprinting.com 